\title{
Under-13 rugby: What are the issues? A panel discussion
}

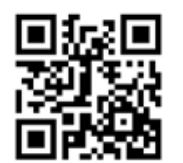

There are many questions about youth rugby for which there are no immediate answers. At what age should the young players specialise? How often should they practise? How many matches should they play in season? Science has lagged behind in providing clear answers, and in many cases decisions about the development of the game at this level have to be made based on logic and intuition. As a result, opinions may differ and controversy may prevail.

Recently a panel of experts representing different aspects of youth rugby was asked a series of questions about this sport, the aim being to examine the answers given from different perspectives. The overall goal was to identify where agreement does or does not exist.

To make this exercise worthwhile, it was important to have respected panellists with expertise in different areas. The following panellists were invited to participate: Justin Durandt, Manager of the Discovery High Performance Centre, Sports Science Institute of South Africa (SA), currently studying towards his $\mathrm{PhD}$ on talent identification in youth rugby players; Dr Sharief Hendricks, a postdoctoral fellow internationally renowned for his research on the tackle in rugby; Dr Mike Marshall, a medical doctor with expertise in rugby medicine and youth rugby; Andries Roux, a rugby coach and teacher working with primary school children; and Dr Eugene Hare, an expert in youth rugby and physical development, and current CEO of the Blue Bulls Rugby Union in SA. The questions posed to each panellist and their individual responses follow.

Should players already start specialising in rugby at U13 age-grade level? What are the potential long-term implications from a health and wellbeing, player performance and player retention point of view if they do specialise early?

Justin Durandt: It is important that we define what we mean by 'specialisation' or 'early specialisation'. I define specialisation as the situation when one engages in intense training in one sport (usually all year round), which results in one not being able to participate in other sports. Early specialisation refers to when this type of specialisation happens while a child is still in primary school. A recent review by Jayanthi et al. ${ }^{[1]}$ in the American Orthopaedic Society of Sports Medicine journal showed that most studies show no benefit of early specialisation in late-specialisation sports (which applies to most team sports, including rugby). On the contrary, they showed benefits for early sampling (playing a number of sports) and only specialising later in high school.

We conducted a study which showed that only a small percentage of boys from U13 Craven Week (24\%) go on to play at U18 Craven Week. ${ }^{[2]}$ Therefore the focus should not be on choosing a small group of elite players at this level, but rather on trying to keep as big a group as possible interested in and playing the game. That is why we should prioritise skill development and participation in primary school and only focus on performance in high school.

This does not mean one cannot have a competitive element, as this is also important, but it must be properly managed not to become the main focus. Talent identification and high-performance programmes should only start in high school at U16 level, when players are physically mature enough to confirm that they have the physical characteristics required for playing rugby at an elite level.
Sharief Hendricks: Short answer, no. At the U13 age-grade level players should be exposed to a variety of sports. With that said, at this stage the player may choose (and key here is the word 'player') to be more competitive in one or two sports, and to be less competitive (i.e. recreational) in other sports. A number of factors may incline this age group towards competitiveness; for example, access to facilities and equipment, social environment and self-efficacy.

Mike Marshall: There are two considerations here: early specialisation within rugby and early specialisation in only rugby. Both have negative implications. Early specialisation within rugby may limit the development of certain skills that may be of value later (e.g. ball skills, positional play, etc.). Also, specialisation prior to 'positiondetermining' growth may limit the development of all-round skills that may well be of benefit later (remember, Springbok lock Eben Etzebeth was a wing while still in his teens!). Early specialisation in only rugby denies the young athlete the opportunity to experience other sports, to develop a wider range of physical and social skills, and to achieve success on a broader front.

Andries Roux: No. I think that they would be better off playing or practising a number of other sports too. This would have a definite influence on their skill set - rugby training can be very restrictive with regard to skill training - and make them more coachable (willing to learn) later on in their careers.

Eugene Hare: In SA, specifically in rugby, the early developers are mainly chosen for provincial sides, but are not always the most talented. A small number of U13 provincial players also play provincial rugby at U16 and U18 level. ${ }^{[2]}$ Positive features of such identification procedures are that children are directed towards sports for which they are physically and psychologically best suited. This in turn means they will probably obtain good results and enjoy their training and participation more.

Another factor is physical injury, which is an inherent risk in sport participation, and to a certain extent must be considered an inevitable cost of athletic training and competition. It is essential that talent in sport be identified at an early stage to make certain that the correct exercise and training are employed to ensure peak performance at a later stage.

How early in the year should U13 age-grade level rugby players start with rugby-specific conditioning and preparation, given that most schools generally have other sporting codes that are also seasonally based?

Justin Durandt: The key to conditioning at a primary school level is to try and achieve general athletic development in children. This means that a school should have a general conditioning programme, irrespective of which sport the child is participating in. Boys only achieve peak height velocity (fastest growth rate) at about 14 years of age, and are usually near their adult height at 18 years of age. This means that many of the physiological traits required for success in elite sport are only evident when children reach the age of 15 - 18 years. Most parents and their children won't know which sport they are best suited to until they are between 15 and 18 years of age. The conditioning programme for the different sports should be coordinated so that there is continuity in conditioning between the winter and summer sports. This will reduce the rugby-specific conditioning period required. The 
children would still require 2 - 3 weeks of rugby-specific activities so that their bodies get used to the contact and sport-specific demands.

Sharief Hendricks: Keeping in mind the previous two questions, the competitive rugby season for the U13 age group should last about 6 months, with a 1-month complete lay-off in-between. In the southern hemisphere this means April through to August. Rugby-specific conditioning and preparation can start 4 weeks prior to the first match. Mike Marshall: Four weeks prior to the first full-contact match might be the ideal time for preseason training in this age group. However, a number of factors make this difficult. Firstly, 4 weeks is probably appropriate for the majority of players in this age group, but perhaps not long enough for those who play at an elite level. Secondly, this 4 -week period must be properly 'periodised' if the injury risk is to be minimised. So, for example, the first week may include only one fitness session, the second week, two sessions of skill and fitness, the third week, three sessions of fitness and skills, and the fourth week, three sessions of fitness and skills plus some contact. Thirdly, there is always a logistical problem in trying to fit in preseason training (e.g. for rugby) during the playing season of another sports code (e.g. cricket). This could be addressed by having a less congested fixture list (see later discussion). Lastly, using the school holidays for preseason training remains a good idea, but is notoriously difficult to achieve due to family commitments, supervision concerns, motivation and so on.

Andries Roux: Two or 3 weeks prior, depending of course on how busy the school's extracurricular programme is and how many of the boys are actually involved in other activities. Having boys choosing to participate in a rigorous rugby preseason instead of, for example, athletics cannot be in their long-term best interest.

Eugene Hare: The SA school sports calendar is a challenging topic. Very little planning is done to accommodate different sports and also include children in various sports activities. A situation analysis with regard to the demands that rugby makes on young rugby players has revealed that the basic skills and abilities a player needs are handling (catching and passing), running, kicking, speed and endurance. Physical abilities required are strength, endurance and flexibility.

The three most common sport activities for boys are rugby, cricket and athletics. Through proper planning the abovementioned demands can be addressed in a training year planner, which can be as follows:

\begin{tabular}{|ll|}
\hline November - December & Active rest \\
\hline January - February & Preseason (Athletics) \\
\hline March & Early season (Rugby) \\
\hline April - May & Midseason (Rugby) \\
\hline June - July & Peak season (Rugby) \\
\hline July - October & Recovery period (Cricket) \\
\hline
\end{tabular}

The challenge for the coach is to draw up a plan for a coaching strategy for the year, a weekly plan, as well as a plan for each session. This will ensure proper player management and the prevention of fatigue.

At U13 age-grade level what should: the maximum length and duration of the rugby season be?; the maximum number of rugby matches played in a season be?; and the maximum number of seasonal matches played per week be? Please elaborate on your answers.

Justin Durandt: The average season, depending on the province and level of the school participating, runs from April to June (12 weeks plus an additional week for Craven Week). In many traditional rugbyplaying schools, boys often play two matches per week, which equates to 24 matches in a 3-month season. This may exclude provincial trials and warm-up provincial games. Rugby is a contact sport (meaning that players require greater periods of recovery compared with non-contact sports) and therefore the number of matches and their frequency should be controlled and monitored. A suggested guideline is a maximum of two games per week (including practice and trials matches) or 25 per season, excluding Craven Week itself. There should be a minimum of a day's break between games, but preferably two. I also think that guidelines are required for primary school tournaments, including the number of games played in a specific time period (days).

Sharief Hendricks: As noted, 6 months with a 1-month complete lay-off in-between. During 1 week, one competitive match (in full kit, etc.) and one practice match. During only 3 of the 4 weeks in a month will competitive matches occur. Every now and then two competitive matches a week may be played. At this level playing the sport (i.e. more game time) is good as it builds the player's capacity to meet the physical demands of the game, and affords the opportunity to execute the technical and tactical skills within a competitive game environment. I would also assume that the player enjoys playing the game. The reason for the regular breaks is to avoid the sport becoming a burden on other aspects of the child's life, and also to allow time to engage in other sports. Players will also most likely be playing contact rugby outside of the formal team setting.

Mike Marshall: I simply don't know what the ideal number of games per season is for this age group, although I would opt for a 'less is more' approach. My 'guesstimate' would be between 10 and 15 matches per season: 10 for the vast majority of 'average players' and 15 for the elite players (i.e. those selected to play for representative teams). I think one game a week, plus perhaps a few extra during a school's rugby festival, is enough for the average player in this age group. In addition, I think no more than a further five or six games should be allowed for the elite players playing for representative teams. Playing more than this in a season means too little time for recovery from 'bumps and bruises' and minor injuries, increasing the risk of more significant injury. Perhaps more importantly, playing more matches than this means too little time for other informal physical activities, schoolwork, hobbies, family time and simple downtime (i.e. mental recovery).

Andries Roux: I think that here the players who go the furthest with the trials or provincial games are at far greater risk. They are the best and most talented players - sometimes just boys who developed fastest - in each age group, and should be looked after. As outlined in my earlier reply, once the trials begin and they begin training with their provincial teams, things get completely out of hand. In 2014 playing time in U13 matches was increased from 20 to 25 minutes per half. I think that this added to the problem. I don't think any one player should be allowed to play more than 25 matches in our (Blue Bulls) season, which already equates to almost two per week. This is going to get complicated in the case of players who are involved in further trials and additional practices.

Eugene Hare: Unfortunately there is no proper benchmark for competitions in all regions in SA. There are also different competition structures that influence when players start to play competitively. The important factors are the number of games per season and the number of games per week. It is recommended that U13 players should not play more than 20 - 25 matches per season (1 000 - 1250 minutes). 
How frequently should players train or practise rugby in a week, in the preseason and in-season periods, at $\mathrm{U} 13$ age-grade level?

Justin Durandt: If they are playing other sports, then one practice session per week during the preseason training would be sufficient. If they are not playing other sports, then two sessions per week could be completed in the preseason. During the season they should have two practice days and one match day a week; if they play a second match in that week this takes the place of one of the practice sessions. Total training load is one of the key principles that needs to be considered when planning practice and game time, and is the minutes that the players practise $\times$ the intensity of each session (on a scale of $1-10$ ).

Sharief Hendricks: Twice a week (this includes the practice match) in the 4 weeks leading up to the first match. The same schedule applies for the in-season period.

Mike Marshall: I think U13 rugby players should train/play three times a week. Preseason training can be done safely three times a week, as long as the training programme is correctly structured/'periodised'. During the season two training sessions and one match per week seem ideal to me, especially if the matches are played on the weekend.

Andries Roux: Preseason: once a week; in season: three times per week (or twice, depending on whether game day falls on a training day). Typically Monday, Tuesday and Thursday should be enough. In Pretoria game days are mostly on Tuesdays and Thursdays.

Eugene Hare: The level of competition will determine the amount of time spent on the abovementioned. Usually primary schools play during the week; therefore the specific game days will determine the amount of training days. It is therefore very important to do the 'hard' and 'tough' training in the preseason and to do specific (specialised) training in the competition phase.

The amount of training in the preseason should therefore be more than during the competition phase: preseason three to four times a week, not exceeding 60 minutes, and peak season two to three times a week, not exceeding 60 minutes.

What is the minimum time U13 age-grade level players need between matches to optimise physical recovery, recover from bumps and bruises, and prepare properly for the next match? Justin Durandt: One day minimum, 2 days is optimal.

Sharief Hendricks: Between 24 and 48 hours.

Mike Marshall: For matches during the 'routine' season, a period of 1 week is required for adequate recovery (both mental and physical). In the less than ideal situation of a school's rugby festival, I think a minimum of 2 days' rest should be enforced between matches.

Andries Roux: At least 2 days.

Eugene Hare: Physical recovery will differ between players and also from game to game. The coach will be the person to determine this. Two days of recovery might be sufficient.

How much contact rugby and contact preparation training (sessions per week, time per session, intensity of contact, preseason, inseason) is enough for optimal rugby coaching and player development at $\mathrm{U} 13$ age-grade level?

Justin Durandt: Contact practices are required to teach contact skills and conditioning. There should be contact in the preseason to prepare the players for in-season matches. The amount and intensity of contact needs to be increased gradually during the preseason. The amount of contact during the in-season may depend on the number of matches in a specific week. If two matches are being played with one practice, you may choose to limit contact in that week. One should always attempt to keep the contact practice as far as possible from the match days, to reduce the likelihood of players having bumps and bruises for their matches.

Sharief Hendricks: Contact is good for preparing players to meet the demands of rugby. In my opinion contact training needs to be considered along a continuum, where you may have controlled lowintensity technical training at the one end (far left), and high-intensity, dynamic match conditions skill training on the other (far right). With this in mind, I think controlled low-intensity technical training can start in week 1 of preseason, with the demands of the contact situation increasing to match conditions up to week 4 . During preseason 50\% of the weekly training schedule should be in preparation for contact. During in-season at least $40 \%$ of the time should be spent on contact technique training. The intensity, level of control and frequency of contact can vary, depending on the goals of that week, what was done in previous weeks, etc. A practice match during the week can form part of this contact training.

Mike Marshall: There is simply no easy answer to this question. The rough and tumble of informal games played by boys of this age group is part of this training, but is obviously unquantifiable! My 'guesstimate' for an adequate rugby programme would be to spend $25 \%$ of time on contact training. So, for example, if there are two 1-hour training sessions per week, 30 minutes of one of those sessions would be a contact session - i.e. specifically match play and rucking practice. Together with the weekly match, that would mean 90 minutes of contact per week during the season. The intensity of the contact training session should be modified to suit the players' fitness level, intensity of the past/pending match, specific training requirements of the team, etc. This contact should be seen as separate from the physical training of specific skills drills like tackling practice, wrestling drills, scrummaging, and so on. As discussed earlier, preseason contact training should only be introduced in the last week of preparation.

Andries Roux: It is necessary to include contact in all training sessions, as it is an invaluable part of any player's skill set. Start as slowly as possible to ensure that everybody understands the skill, before lifting the tempo towards the level that you expect during games. In-season, contact should be kept to a minimum. Each and every training session should include aspects of every skill required: physical abilities like agility, strength and speed, ball skills like catching and passing, contact skills like tackling and rucking, etc.

Eugene Hare: Contact at primary school level is not as intense as in senior rugby. Therefore an analysis of the game should give more accurate statistics of the game and specifically the contact situation. Different defensive structures will determine which players (position in the team and in defence structures) will make the most tackles. This might differ from senior rugby. Contact confidence skills training should be part of preseason training and at all sessions, and be lowered in-season.

How does one maintain sufficient balance between rugby and other important developmental aspects of life in U13 age-grade level rugby players?

Justin Durandt: One needs to educate both parents and teachers. Many schools are hiring private coaches who are judged on the team's results, and therefore they have a 'win at all costs' attitude. 
We need to strive to achieve three goals with children playing rugby and any sport: personal development, participation and performance. ${ }^{[3]}$ The key focus at primary school should be to create opportunities for participation and personal development.

Sharief Hendricks: I think the roles and responsibilities and the relationship between the coaches, parents and players are primarily to find the optimal balance. Also, unnecessary pressure on the player should be avoided. In the same vein, excessively reinforcing rugby achievements may give the player a false sense of ability, which may affect him/her later in life.

Mike Marshall: This is the essence of this panel discussion, i.e. maintaining a balance between rugby and all the other developmental aspects of a child's life is crucial. Unfortunately there are no easy solutions to this matter, as it is complicated by numerous factors. These include, but are not limited, to:

- Time constraints (e.g. a busy school/academic programme, more extracurricular school responsibilities than ever before, participation in other sports codes, busy family life, etc.)

- Logistic difficulties (e.g. too few coaches, inadequately skilled coaches, inadequate school sports facilities, etc.)

- Pressure to succeed (e.g. misguided parents, scholarships and bursaries, ambitious coaches)

- Age (e.g. mental and physical immaturity).

The (utopian) solution lies in providing a structured approach to exercise, where the needs and desires of each individual are met. Of course this is just about impossible to provide, so perhaps a more utilitarian approach would include the following guidelines:

Children in this age group should be encouraged to play a different sports code during each of the four school terms. This would have the beneficial effects of:

- necessitating a shorter rugby season (see earlier discussion on this topic)

- de-emphasising any one particular sports code

- facilitating better all-round physical development of the child.

Parents and teachers must insist on the provision of sufficient time for homework, assignments, etc. Social interaction around the child's sports participation should be encouraged.

Andries Roux: This is very difficult. Parents are guided by the whims of coaches and teachers. Coaches earn more the more they coach, and teachers are sometimes not necessarily specialists in their field. There are no clear guides as to what should be done and how it should be implemented. Short-term success sometimes gets in the way of common sense. The only way to find a balance is by coordinating the entire school's extracurricular programme. I think that it is important that a team of experts provides clear guidelines, keeping in mind that these activities form a part of a learner's whole education and should not be detrimental towards other aspects. I think for starters there should be a date on which a preseason is allowed to start. There should also be guides as to how many training sessions should be allowed per week, etc. Educating the coaches is going to be very important.

Eugene Hare: Player management is the key to success. If the player is identified as talented in a specific sport, then proper planning and management can be executed. Career planning can be done and growth and development can determine the players' long-term development.
How much rugby played is enough to maintain passion and enthusiasm for the game, for long-term player retention, and for effective long-term player development pathways for $\mathrm{U} 13$ rugby players wanting to play rugby at the elite adult level?

Justin Durandt: We need to study the occurrence of injuries at primary school level and come up with definitive guidelines on training and match time in relation to injury risk.

Sharief Hendricks: Côtés Developmental Model for Sport Participation ${ }^{[3]}$ and biopsychosocial approach serve as good reference points to answer this question. In these models the goals and objectives of the individual set the course for recreational participation or elite performance. With this in mind, if a player enjoys playing rugby and wants to play and train more (with no outside pressures), then he should be fully supported by providing the optimal environment in order to afford the player this opportunity.

Mike Marshall: The 'balance of activities' argued for in the questions above is the way to maintain long-term sports participation. When any sports participation becomes onerous and is no longer fun, long-term participation is most unlikely. In addition, the development of better 'allround' motor skills can only be of benefit to the growing rugby player.

Andries Roux: This is also a tricky problem because some parents believe in keeping their kids busy, so the moment you train less some parents are just going to fill the gap with another activity.

I have often wondered if changing the matches/competitions to include some other physical activities isn't the solution. By making games/matches shorter and including other competitions to eventually determine the result, one would be encouraging coaches to focus on more than just game-plans, moves and set patterns. For example, the game is played with two 12/15-minute halves. All the boys then participate in an activity or two against each other. Whether they compete in $40 \mathrm{~m}$ sprints, do push-ups, run ladders, bridges, do hand-eye coordination drills, kick, or catch up-and-unders, the results of these 'games' would then also count towards the result on the day. This would encourage coaches to include a wider range of physical exercises/activities in their training.

I think that a programme that addresses these physical abilities would benefit other sporting codes. They could adopt a similar model in their programmes. This in turn could result in less sport-specific coaching and take the emphasis off the 'win at all costs' mentality that is currently such a serious concern. Children would learn through practising a wider range of skills on a regular basis. I think that by doing this one would actually be improving the skill sets of the players immeasurably.

Eugene Hare: Proper scientific research is needed to ensure that the amount of rugby played by 13-year-old children supports their growth and development as well as other physiological and social development. The length of the season, amount of games, length of active rest and participation in other sports will be influential factors.

\section{References}

1. Jayanthi N, Pinkham C, Dugas L, Patrick B, LaBella C. Sports specialization in young athletes: Evidence-based recommendations. Sports Health 2013;5(3):251-257. [http:// dx.doi.org/10.1177/1941738112464626]

2. Durandt J, Parker Z, Masimla H, Lambert MM. Rugby-playing history at the national U13 level and subsequent participation at the national U16 and U18 rugby tournaments. S Afr J Sports Med 2011;23(4):103-105.

3. Côté J, Hancock DJ. Evidence-based policies for youth sport programmes. Int J Sport Policy Politics 2014; Jun 26:1-15. [http://dx.doi.org/10.1080/19406940.2014.919338]

S Afr J Sports Med 2015;27(3):63-66. DOI:10.7196/SAJSM.8078 